\title{
Rétroactions entre la dynamique sableuse de la plage et l'efficacité des ganivelles pour la protection et le développement du cordon dunaire de Torreilles (66)
}

\section{Mathieu GERVAIS ${ }^{\mathbf{1}}$, Philippe RICHARD ${ }^{\mathbf{1}}$, Hugues HEURTEFEUX ${ }^{\mathbf{1}}$, Delphine BOULET ${ }^{1}$, Michel MOUYSSET ${ }^{1}$, Pierre LOUBET ${ }^{1}$, Stéphanie GAUTIER ${ }^{2}$, Provence LANZELLOTTI ${ }^{3}$}

1. EID Méditerranée, Pôle littoral, 165 avenue Paul Rimbaud, 34000 Montpellier, France.mgervais@eid-med.org;prichard@eid-med.org

2. Perpignan Méditerranée Métropole, 11 boulevard Saint-Assiscle, BP 20641 , 66006 Perpignan Cedex, France.

s.gautier@perpignan-mediterranee.org

3. Agence d'Urbanisme Catalane Pyrénées Méditerranée, 19 espace Méditerranée, 66000 Perpignan, France.

provence.lanzellotti@aurca.org

\section{Résumé :}

Sur beaucoup de systèmes dunaires méditerranéens qui ont été détériorés par l'urbanisation, certains sites présentent encore des dunes préservées ou restaurées. L'intérêt de la mise en défens et des ouvrages à effet brise-vent type ganivelles est incontestable pour une remise en état des dunes. Pourtant, la littérature traite peu du lien entre l'efficacité de ces interventions et la morphodynamique de la plage dans son ensemble. Cette contribution présente le bilan d'un travail de 5 années de suivi du système plage/dune de Torreilles, suite à la mise en place d'ouvrages de ce type en 2014, travaux commandés par Perpignan Méditerranée Métropole. L'originalité de ce travail est de relier la dynamique du front dunaire aux variations longitudinales de largeur de plage et aux mouvements des grandes sinuosités liées aux barres d'avant-côte en festons, d'après un suivi de haute résolution au DGPS, adapté aux morphologies rencontrées.

Les résultats prouvent l'efficacité de ces équipements pour piéger le sable et favoriser la reprise de la couverture végétale. Le dépôt dans les ganivelles est manifestement plus fort sur la zone naturelle du site où la largeur de plage est importante. En Méditerranée microtidale, ce critère parait fondamental pour la réussite d'un aménagement. Sur les sites en érosion, étroits, avec des pentes de plages plus fortes et aucune berme, ni haut de plage, le jet de rive est plus haut; les risques de processus de collision apparaissent plus élevés. Mots-clés :

Ganivelles, Transport éolien, Erosion, Tempête, Rétroaction morphologique, Morphodynamique, Barres d'avant-côte, Run-up des vagues, Jet-de-rive. 


\section{Thème 2 - Dynamique sédimentaire}

\section{Introduction}

En France, 16500 bâtiments sont directement menacés par l'érosion aujourd'hui, pour une valeur de 3,7 milliards d'euros (CEREMA, 2019). Si beaucoup de systèmes dunaires ont été dégradés en Méditerranée par l'urbanisation, la mise en défens par un système de clôture canalisant les accès, et des méthodes douces de piégeage sableux sur la dune vive ont permis d'améliorer visiblement leur état de santé ces 30 dernières années. Les volumes perdus ont pu se recréer et la végétation reprend sur les anciens cheminements anarchiques, ce qui prouve la capacité de cicatrisation des dunes.

La végétation et son développement face à l'apport éolien reste l'élément inconditionnel de la croissance et de la fixation des dunes bordières littorales. Or, le golfe du Lion est marqué par un antagonisme fort des directions de vents dominants, marins et surtout terrestres ; les dunes y sont moins développées en hauteur et en largeur que sur les autres côtes françaises (HEURTEFEUX \& RICHARD, 2005). Il faut ajouter à cela l'étroitesse des plages, en l'absence d'estran de marée, ainsi que de fortes sécheresses qui limitent le développement des plantes.

Afin de "donner un coup de pouce" à la nature et favoriser le processus éolien de piégeage sableux liés aux plantes, les ganivelles sont devenues l'outil clef de l'aménagement dunaire. Cette technique, utilisant des matériaux durables, est apparue dans les années 1980 dans la région, grâce à l'impulsion de l'EID, en suivant l'exemple du savoir-faire développé sur la côte atlantique, en Aquitaine notamment. Du point de vue littérature, SABATIER et al. (2009) proposent une synthèse intersite intéressante, et il existe d'autres retours d'expériences dans des ouvrages de référence, notamment GOUGUET (2018).

Il est évident aujourd'hui que les impacts des tempêtes, qui conditionnent la réussite d'un projet d'aménagement, sont dépendants de la morphodynamique des barres d'avant-côte en festons (CASTELLE et al., 2015). En dehors des épisodes de collision des vagues de tempête, le taux de submersion de la plage et l'orientation précise du vent jouent un rôle essentiel (HAGE et al., 2018), davantage que la force et la fréquence du vent marin luimême. A ce titre, les épisodes de rechargement éolien efficace de la dune sont généralement très brefs en Méditerranée (quelques dizaines d'heures par an), puisque les épisodes de vent marin sont le plus souvent associés à des pluies et des submersions qui bloquent les mouvements éoliens.

\section{Site d'étude et méthodologie}

Différents secteurs du linéaire côtier de Torreilles ont été étudiés grâce à un suivi DGPS centimétrique : un secteur naturel et un secteur urbain en érosion, et des systèmes naturels de plages plus larges et plus stables (figure 1). L'objectif principal était de réaliser des MNT de très haute résolution de la dune, de la plage et des systèmes de barres d'avantcôte. De très nombreuses radiales topographiques ont été réalisées entre les lignes de ganivelles afin de rendre compte de leur potentiel ensablement. 


\section{XVI İmes Journées Nationales Génie Côtier - Génie Civil \\ Le Havre 2020}

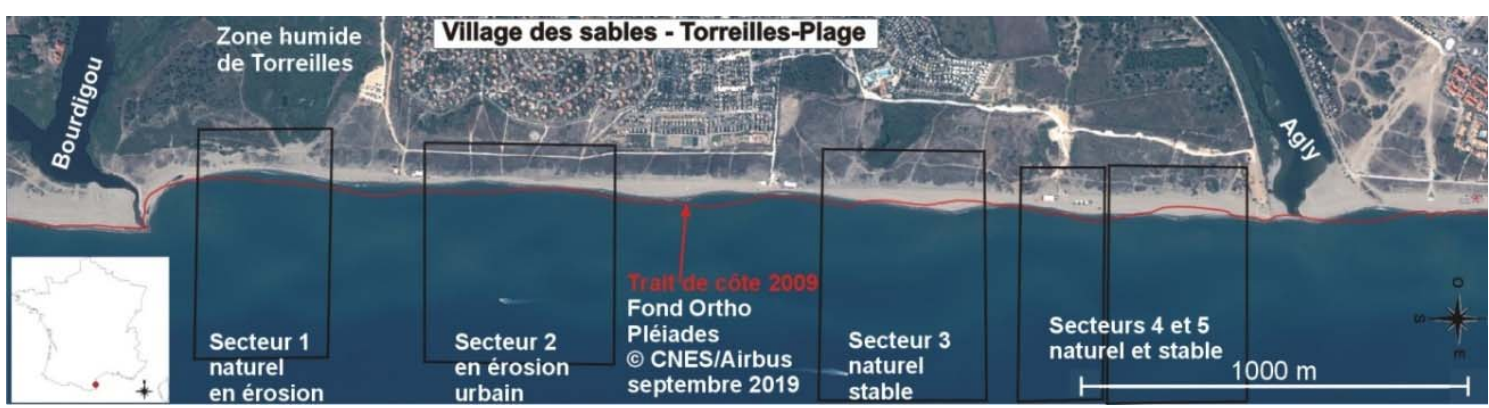

Figure 1. Le site d'étude de Torreilles au sud du Golfe du Lion et les 5 secteurs de plage contrastés suivis, et évolution du trait de côte sur 10 ans.

Comme le montre la figure 1, le site de Torreilles présente un trait de côte avec de fortes sinuosités de plage, formant de grands motifs de croissants rythmiques reflétant la position des barres d'avant-côte festonnées, et en particulier de la barre interne. La barre externe festonnée est une structure très stable de $700 \mathrm{~m}$ de longueur d'onde située entre -5,5 et -6 m. Elle ne bouge que faiblement sur plusieurs années (déplacement max. d'environ 50 m entre 2011 et 2019), elle n'est donc pas couverte par notre analyse. La barre interne est une structure complexe et dynamique constituée d'une imbrication de festons, de barres transverses et chenaux s'enracinant au bord. Elle change de forme chaque hiver, principalement lors des fortes tempêtes qui peuvent venir d'une direction très oblique $(\mathrm{S}-) \mathrm{SE}$, ou très frontale depuis l'E (angle d'incidence des vagues de tempête de $\mathrm{Hs}>3 \mathrm{~m}$ au large : entre $+60^{\circ}$ et $-10^{\circ}$ à $40 \mathrm{~m}$ de profondeur, d'après la bouée de Leucate voisine). La dérive littorale est importante $\left(\sim 40000 \mathrm{~m}^{3} / \mathrm{an}\right)$ et dirigée vers le nord. Elle provoque le déplacement d'environ $80 \mathrm{~m}$ vers le nord des morphologies rythmiques de la barre interne, et par effet de rétroaction, des sinuosités de la plage : position des creux et des avancées du trait de côte (caps). Cela explique que la partie sud du site soit fortement impactée par l'érosion, en raison du blocage de la dérive par la digue d'embouchure du Bourdigou. En moyenne, la largeur de plage en été avoisine $40 \mathrm{~m}$ sur les secteurs 3-4-5 au nord, et $20 \mathrm{~m}$ sur les secteurs 1 et 2 au sud. Le pied de dune de Torreilles se situe actuellement autour de $+2,5 \mathrm{~m} \mathrm{NGF}$, et la crête de dune varie entre $+3 \mathrm{~m}$ et $+4,5 \mathrm{~m}$ NGF.

\section{Résultats}

\subsection{La dynamique générale du site sur 5 ans}

Une analyse préliminaire de la dynamique du site de Torreilles sur une échelle de temps plus longue, basée sur des images aériennes et des levés lidar, a révélé que les impacts sur le front dunaire des fortes tempêtes $(\mathrm{Hs}>5 \mathrm{~m})$ des 10 dernières années ont systématiquement été localisés dans les zones de creux des sinuosités du trait de côte, jamais sur les zones d'avancées. Il s'avère donc que la largeur de plage est un facteur morphologique critique pour le régime de submersion et de collision par les vagues. Il est 


\section{Thème 2 - Dynamique sédimentaire}

en première approche supposé que, comme vu sur d'autres sites du golfe du Lion, des effets de découplage syn-tempête se produisent entre la plage et les barres, sur l'axe longitudinal, et que cela puisse être le phénomène à l'origine des impacts dans la dune (voir BALOUIN et al., 2013).

Au cours de la période de suivi 2014-2019, plusieurs hivers très contrastés en termes de houles se sont suivis. La figure 2 présente le houlogramme, avec une estimation grossière des hauteurs maximales de run-up atteintes sur la plage lors des principaux évènements (en rouge), selon les paramètres de houle mesurés au large et la formule de STOCKDON et al. (2006), intégrant la pente de la plage de Torreilles, et en prenant en compte les niveaux de surcotes enregistrés par le marégraphe de Port-la-Nouvelle.

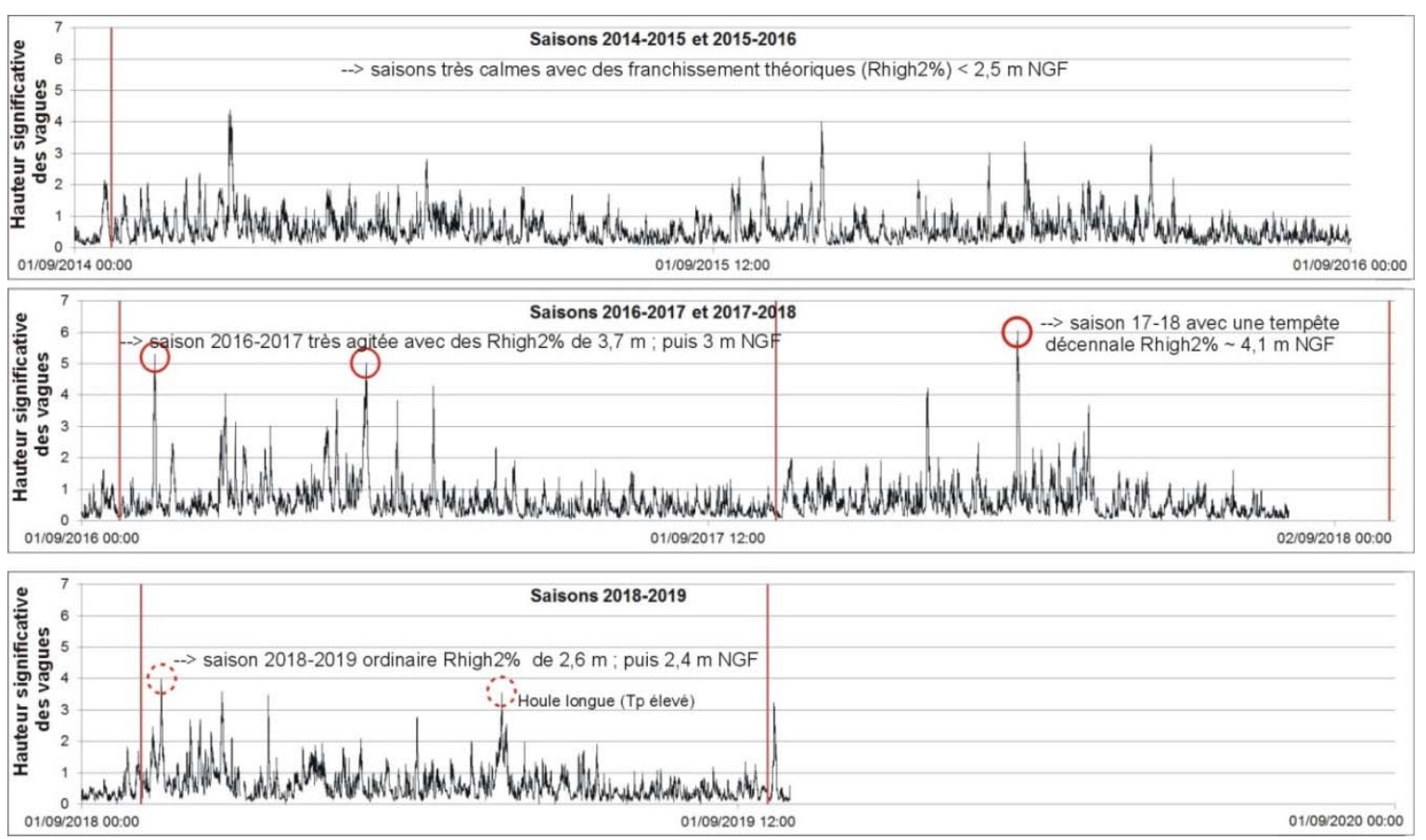

Figure 2. Houlogramme de la Hs des vagues à la bouée de Leucate située à $20 \mathrm{~km} \mathrm{au}$ nord du site (40 m de fond); dates des levés représentés par des barres rouges, et commentaires sur l'élévation estimée du jet de rive des principales tempêtes.

Malgré certains hivers plutôt "calmes", à chaque saison des dégâts ont été constatés sur le front dunaire de la partie sud du site. L'hiver 2016-2017 a été particulièrement riche en tempêtes et a laissé de nombreux dégâts, avec un effet très morphogène pour la plage, tandis que l'hiver 2017-2018 a vu une tempête décennale le 01/03. Celle-ci a bouleversé l'ensemble de la dune avec un franchissement généralisé par les vagues, provoquant des overwashes et un blanchiment sableux de la dune fixée (voir une illustration en figure 3). La dernière saison présente un hiver plus classique du point de vue de l'énergie des vagues, mais ses épisodes de taille moyenne $(\mathrm{Hs} \leq 4 \mathrm{~m})$ ont généré toutefois des dégâts marqués au sud. 


\section{XVI èmes Journées Nationales Génie Côtier - Génie Civil \\ Le Havre 2020}

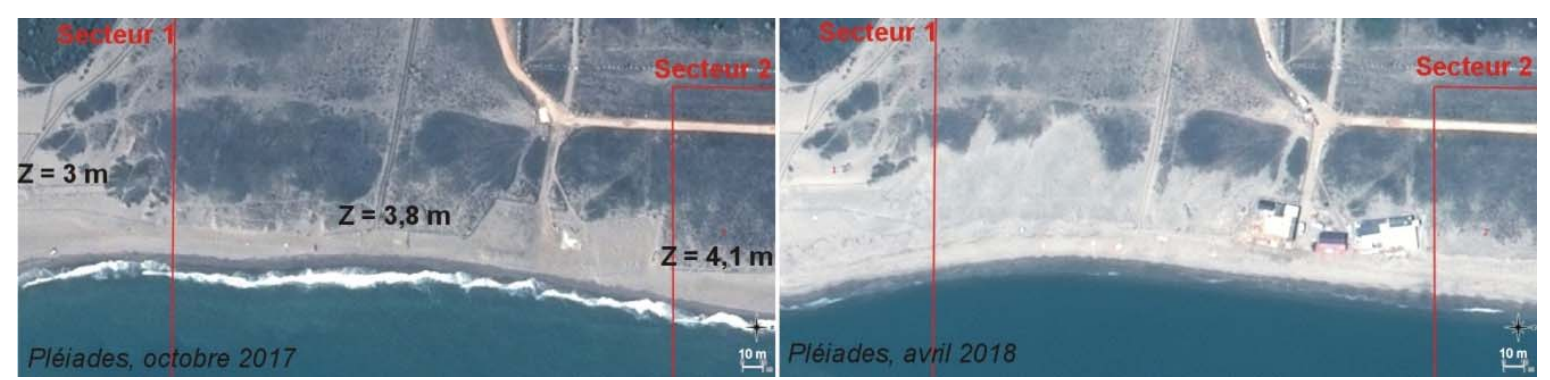

Figure 3. Illustration sur des images satellites THRS Pléiades (ㄷ CNES/Airbus/IGN) de l'ensablement généralisé de la dune fixée après la tempête du 01/03/2018 ; l'élévation initiale de la crête de dune est indiquée sur l'image de gauche.

Des volumes ont été calculés sur chacun des secteurs en regroupant les 4 et 5 , contigus au nord : émergé (au-dessus de l'élévation $0 \mathrm{~m} \mathrm{NGF}$ ) et immergé (barres internes, en dessous ce niveau).

Les résultats montrent que l'érosion domine très largement sur les petits fonds; cependant les secteurs les plus en érosion ne sont pas forcément ceux du sud. Sur la plage émergée, les courbes de la figure 4 indiquent que les secteurs 1 et 2 sont en forte érosion, perdant plusieurs milliers de $\mathrm{m}^{3}$ de sable chaque année. Au contraire, le secteur 3 en gagne un peu, surtout en 2018-2019 (en raison de l'accolement d'une barre à la plage ; ce gain est probablement temporaire). Enfin, malgré des modifications de formes importantes sur les plages et une forte variabilité inter-annuelle de leurs volumes, le bilan global des secteurs 4 et 5 reste stable. Les secteurs nord de Torreilles semblent donc nettement plus résilients face aux coups de mer que les secteurs sud. Par exemple, lors de la saison de la tempête décennale de mars 2018, la majorité des plages sont en érosion, mais cette érosion est inversée la saison suivante sur les secteurs au nord, à l'inverse des secteurs au sud. La plage émergée du sud s'érode donc de manière chronique, depuis 2016 sur le secteur 1, et depuis 2017 sur le secteur 2.

\subsection{Le lien entre les dunes et la contrainte imposée par la largeur de plage}

En dehors du cas des fortes tempêtes qui provoquent des impacts dans la dune, on observe une déconnexion du comportement de la plage et de celui de la dune : celle-ci peut s'engraisser sous l'action des vents alors que la plage s'érode sous l'action des vagues. Ce phénomène d'accumulation éolienne est particulièrement clair sur les secteurs au nord de Torreilles, à large plage et sans laisse de mer ou trace de collision par les vagues observées (excepté lors de la tempête de mars 2018 et quelques modifications faibles lors de l'hiver 2016-2017). 


\section{Thème 2 - Dynamique sédimentaire}
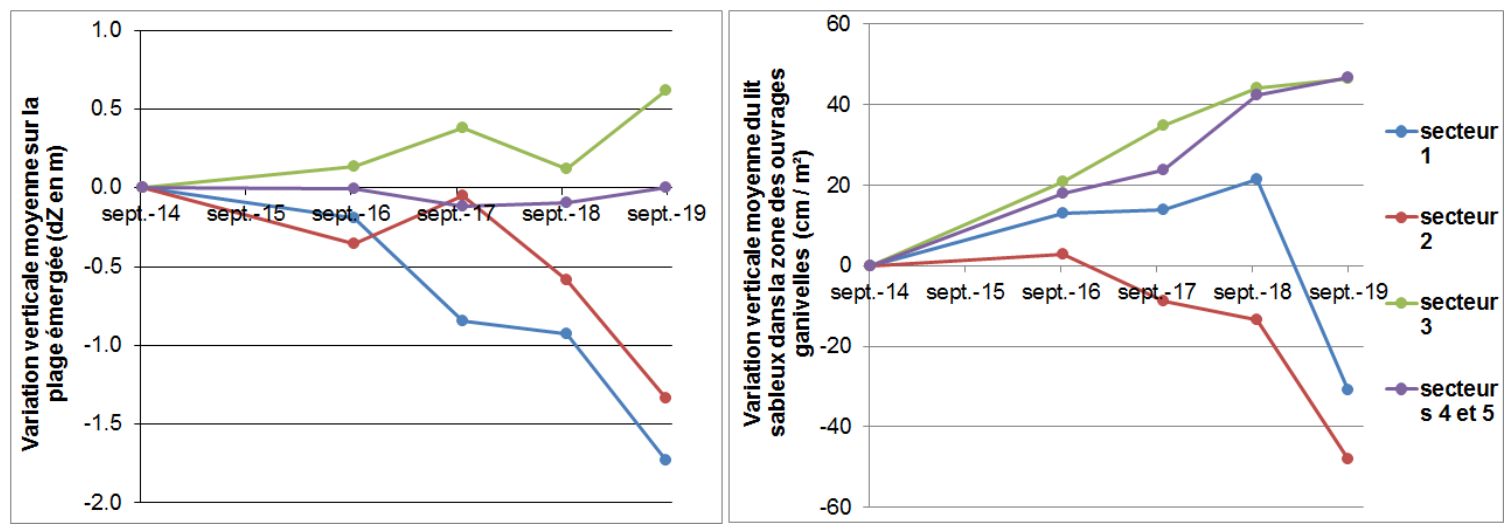

Figure 4. Evolution temporelle par secteur de la variation verticale du niveau moyen de la plage (à gauche) et de la zone au sein des ouvrages de protection dunaire (à droite;

N.B. : variations pondérées par la surface des secteurs).

La figure 4 montre l'ensablement régulier des ouvrages sous l'action des vents marins (jusqu'à $10 \mathrm{~cm} / \mathrm{an}$ ). Au sud, des impacts se produisent sur le front dunaire ce qui traduit les phases d'érosion depuis 2016 (secteur 2) et 2017 (secteur 1). L'accumulation entre 2017 et 2018 représente partout l'action du franchissement par les vagues lors de la tempête majeure qui, étonnamment, a provoqué un dépôt de sable sur le devant de la dune et entre les ganivelles, sans détruire les ouvrages. L'exception est dans ce cas le secteur 2 , et le nord du secteur 1 non suivi, où les ganivelles ont été détruites en même temps que le front dunaire s'est creusé.

\subsection{Rétroactions barres-plage-dune}

Les impacts dans la dune sont fortement influencés par le critère de largeur de plage entre le nord et le sud (actuellement $10 \mathrm{~m}$ de large seulement). Aussi, la forme de profil émergé est bien différente : la pente est plus raide au sud, avec une absence de haut de plage, qui forme d'habitude un plateau très typique sur les plages locales. En conséquent, le jet de rive de tempête n'interagit plus au sud avec un espace tampon d'infiltration avant la dune. Des observations en conditions agitées montrent que le run-up des vagues est plus élevé et atteint plus facilement le pied de la dune au sud du site. L'hypothèse d'impacts plus forts au sud, en lien direct avec la dynamique des barres et les creusements des sinuosités (rétroaction profil émergé/immergé) semble ici assez évidente. L'hypothèse proposée est que, comme indiqué par des flèches sur la figure 5, la circulation $2 \mathrm{D}-\mathrm{H}$ des courants induits par les vagues sur les barres festonnées/transverses en pied de plage, empiète davantage au sud qu'au nord sur la zone habituelle du jet de rive de tempête sur la plage, le swash emportant davantage de sable en fin d'épisode de tempête. Ce remodelage monte ici jusqu'en pied de dune. 


\section{XVI èmes Journées Nationales Génie Côtier - Génie Civil \\ Le Havre 2020}

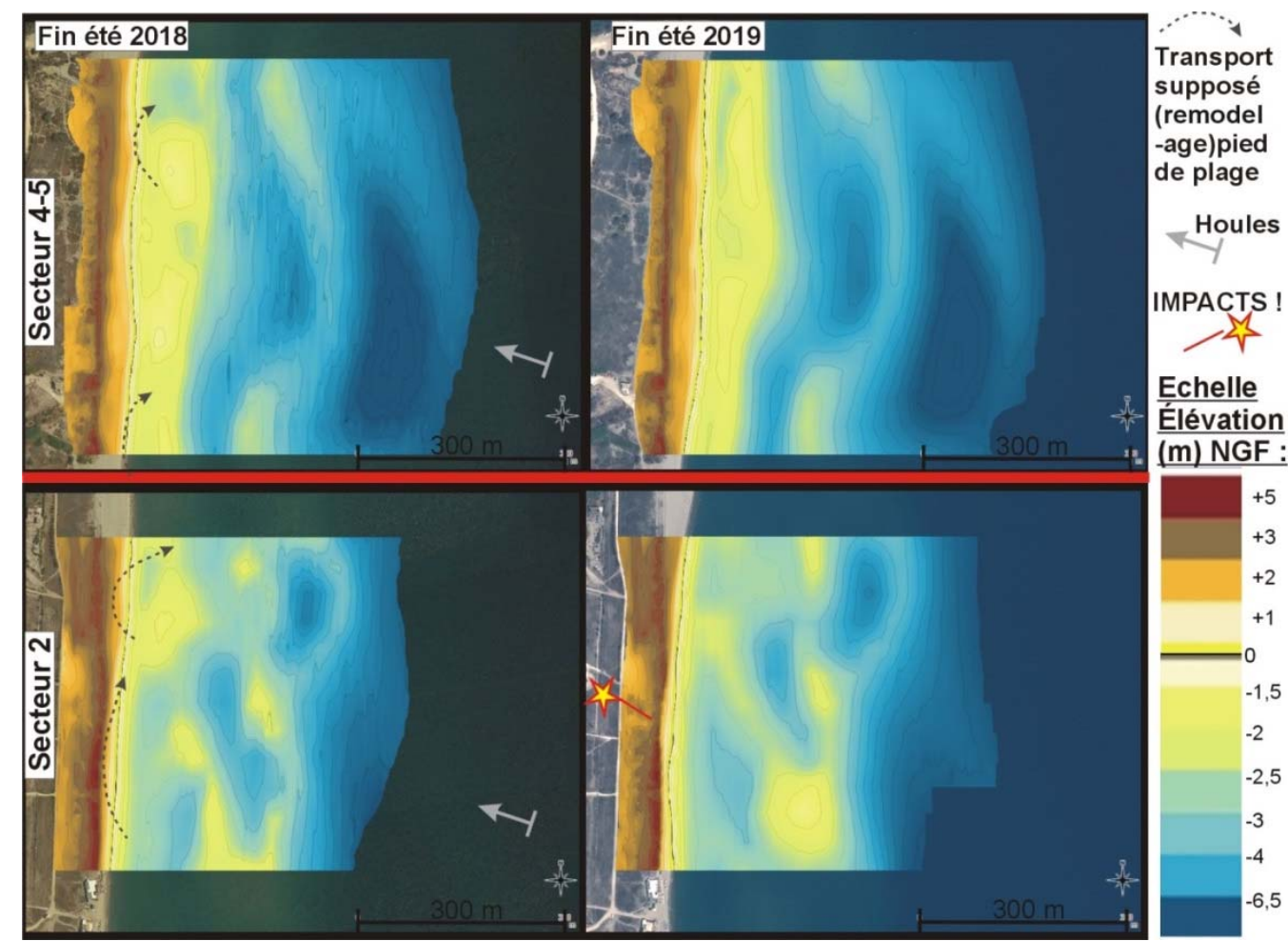

Figure 5. Comparaison de l'évolution morphologique entre les étés 2018 et 2019 sur le secteur 2 au sud, et les secteurs 4-5 au nord de Torreilles.

\section{Conclusion}

Les résultats prouvent l'efficacité des lignes et casiers de ganivelles pour piéger le sable. Néanmoins, le dépôt dans les ouvrages est manifestement plus visible sur la zone naturelle de Torreilles où la largeur de plage est importante. En l'absence de marée, en Méditerranée, ce critère est fondamental pour que l'aménagement soit une réussite. Les secteurs de plage très étroits et en érosion ne vont pas profiter du rechargement par les vents de mer et vont subir de manière préférentielle des impacts dus à l'uprush des vagues. Il est observé que les ouvrages en pied de dune peuvent soutenir l'impact d'une tempête décennale, malgré un franchissement total du cordon, à condition d'être correctement implantés et suffisamment en recul pour moins subir l'impact direct des vagues. Sur les sites étroits, dans les creux des sinuosités de plage liés aux barres, on observe des régimes de collision plus fréquents sur le front dunaire, détruisant les ouvrages et réduisant à néant leurs bénéfices. Sur les sites en érosion, avec des pentes de plages plus fortes et sans plateau de haut de plage, le jet de rive est manifestement plus énergétique et profite d'une rampe d'accès directe vers la dune, augmentant, selon nos observations, la hauteur du run-up et le remodelage des croissants, en lien avec le mouvement des barres. Ce point important nous incite à repenser les aménagements en acceptant un recul des ouvrages de protection dunaire, pour une durée de vie accrue. 


\section{Thème 2 - Dynamique sédimentaire}

\section{Remerciements}

L'EID Méditerranée remercie l'agglomération de Perpignan Méditerranée et l'Agence de l'Eau Rhône-Méditerranée-Corse d'avoir financé ce suivi de plusieurs années.

\section{Références bibliographiques}

BALOUIN Y., TESSON J., GERVAIS M. (2013). Cuspate shoreline relationship with nearshore bar dynamics during storm events - field observations at Sete beach, France. Journal of coastal research, Special Issue No. 65, pp 440-445, https://doi.org/10.2112/SI65-075.1 CASTELLE B., MARIEU V., BUJAN S., SPLINTER K.D., ROBINET A., SENECHAL N., FERREIRA S. (2015). Impact of the winter 2013-2014 series of severe Western Europe storms on a double-barred sandy coast: Beach and dune erosion and megacusp embayments. Geomorphology 238, pp 135-148. https://doi.org/10.1016/j.geomorph.2015.03.006 CEREMA (2019). Évaluation des enjeux potentiellement atteints par le recul du trait de côte. https://www.cerema.fr/fr/system/files?file=documents/2019/10/evaluation_enjeux_potentiellement_atteints_p art_recul_trait_cote_v_octobre_2019.pdf

GOUGUET L. (2018). Guide de gestion des dunes et des plages associées. Editions Quae, 215 p. https://doi.org/10.35690/978-2-7592-2482-1

HAGE P., RUESSINK G., DONKER J. (2018). Using Argus Video Monitoring to Determine Limiting Factors of Aeolian Sand Transport on a Narrow Beach. Journal of Marine Sicence and Engineering Vol. 6(4), 138, 17 p. https://doi.org/10.3390/jmse6040138 HEURTEFEUX H., RICHARD P. (2005). Vingt ans de protection des dunes littorales par des techniques douces : bilan et perspectives à travers quelques exemples en Méditerranée française, Milieux littoraux, nouvelles perspectives d'étude, Paris, Harmattan, pp 143-166

SABATIER F., ANTHONY E.J., HEQUETTE A., SUANEZ S., MUSEREAU J., RUZ M.H., REGNAULD H. (2009). Morphodynamics of beach/dune systems: examples from the coast of France. Géomorphologie : relief, processus, environnement, Groupe français de géomorphologie (GFG), Vol. 15(1), pp 3-22. https://doi.org/10.4000/geomorphologie. 7461

STOCKDON H.F., HOLMAN R.A., HOWD P.A., SALLENGER A.H. (2006). Empirical parameterization of setup, swash, and runup. Coastal Engineering, Vol. 53, pp 573-588. https://doi.org/10.1016/j.coastaleng.2005.12.005 